\section{Why a benign ovarian mass can still undergo surgery by a} gynaecological cancer surgeon?

\author{
M. Quaranta ${ }^{1}$, G. Mehra ${ }^{1,2}$, R. Nath ${ }^{1}$, A. Sayasneh ${ }^{1,2}$ \\ ${ }^{1}$ Department of Gynaecological Oncology Guy's and St Thomas' NHS \\ Foundation Trust; ${ }^{2}$ Faculty of Life Sciences and Medicine King's College \\ London
}

Objectives: Overtreatment of benign ovarian masses has significant health implications and socio-economic costs. We reviewed the MDT outcome, imaging and histological characteristics as well as operating details of the masses falsely presumed malignant who underwent surgical treatment by the gynaecological oncology team.

Methods: Prospective single centre cohort study that included 182 patients from February 2017 till February 2018 with adnexal masses that were preoperatively defined as malignant by our MDM and received surgical treatment. We assessed MRI findings and ADNEX score as well as final histology, operative details and surgical complications.

Results: 33 masses (18\%) were benign. Of these $11(33.3 \%)$ were mucinous, 10 (30.3\%) serous cystadenomas and $12(36.4 \%)$ constituted other miscellaneous types. MDT discussion and MRI-based imaging preoperatively diagnosed 16 masses (48.5\%) as borderline and $17(51.5 \%)$ as malignant. Retrospective application of the ADNEX model on stored images of 24 available cases calculated relative risk and defined 3 categories as follows: BOT 15 (62.5\%), 4 benign (16.6\%), 5 metastatic (20.8\%). The majority (82\% patients had an open procedure and 32 of these were over treated with radical surgery. Average operating time and LOS were $164 \mathrm{~min}$ and 5 days respectively. We report 6 cases of postoperative $\mathrm{CD} 2$ complications and 1 intraoperative small bowel injury.

Conclusions: The epidemic of overtreatment has significant costs in terms of impact on womens' health and on the resources of the health system. The ADNEX model may improve our pre-operative diagnosis.

References:

1 Dindo D, Demartines N, Clavien P-A. Classification of surgical complications. Ann Surg. 2004; 240(2): 205-213.

2 Van Calster et al. Evaluating the risk of ovarian cancer before surgery using the ADNEX model to differentiate between benign, borderline, early and advanced stage invasive and secondary metastatic tumors: prospective multicentre diagnostic study. BMJ. 2014; 349.

\section{Fusion imaging as a promising technique to locate uncertain PET- CT capitation}

\section{Chiappa, G. Bogani, F. Raspagliesi}

Gynaecologic Oncology, National Cancer Institute of Milan, Milan, Italy

Objective: To propose fusion imaging in the preoperative evaluation of patients affected by gynaecological cancers. A multidisciplinary approach is extremely important in the pre-operative workup of gynaecological cancers; fusion imaging allows a direct comparison between ultrasound and other imaging techniques (CT-scan, MRI, PET-CT).

Methods: All patients with gynaecological malignancies underwent pre-operative ultrasound (US) for disease evaluation and staging. All other imaging exams of the patient were imported into the US machine and synchronized with US through the Navigation system. This allowed the examiner to navigate through CT and PET volumes while moving endocavitary or transabdominal probe.

Results: This methodology allowed us to verify prior to surgery many challenging questions; in particular we can overlap PET-CT images and ultrasound with the possibility to localize uncertain PET-CT captation, thus distinguishing between ovarian activity in young patients, lymph nodes, peritoneal nodules of carcinomatosis. We present four cases of patients in which fusion imaging between PET-CT and ultrasound helped in preoperative patients' workup. In the first case an uncertain PET-CT captation was referred to ovarian activity in a young patient. In the second case PET-CT showed pelvic captation in a patient treated with surgery and first line chemotherapy for a serous high grade ovarian cancer. Fusion imaging allowed us to identify peritoneal nodules at ultrasound corresponding to the recurrence identified at PET. During surgical exploration, with intraoperative ultrasound, we could then help the surgeon to identify the recurrence. In the third case PET-CT described a pelvic nodule in a patients with previous ovarian cancer; with Fusion imaging we could locate the nodule corresponding to PET captation and we could better describe the recurrence as a nodule infiltrating rectosigmoid wall up to the muscular layer. In the last patient we could overlap omental cake at PET-CT with ultrasound to identify where to perform a tru-cut biopsy in a case ovarian cancer before NACHT.

Conclusions: Fusion imaging could be considered an important tool in the preoperative patients workup in an Oncological referral Centre, to promote and consolidate a multidisciplinary team and to better locate uncertain PET-CT captation, especially in young patients with ovarian activity or in cases of recurrent disease.

\section{Intraoperative ultrasound in the conservative treatment of borderline ovarian tumors}

\author{
V. Chiappa ${ }^{1}$, G. Murru ${ }^{1,2}$, G. Bogani ${ }^{1}$, V. Galiano ${ }^{1,2}$, A. Ditto ${ }^{1}$, \\ F. Martinelli ${ }^{1}$, D. Vinti $^{1,3}$, F. Raspagliesi ${ }^{1}$
}

${ }^{1}$ Gynecologic Oncology, National cancer Institute of Milan, Milan, Italy; ${ }^{2}$ Department of Obstetrics and Gynaecology, ASST S.Paolo and Carlo, San Paolo Hospital, Milan, Italy; ${ }^{3}$ Department of Obstetrics and Gynaecology, Mangiagalli Hospital, University of Milan, Milan, Italy

Objectives: Borderline ovarian tumors have an excellent prognosis, so conservative surgery can be proposed and it's safe in young patients. However, the recurrence rate described in literature is $5-56 \%$ and small lesions can be detected in the ovary at ultrasound but hardly recognizable during surgical exploration. We propose an ultrasound-guided surgery technique to allow the enucleation of the tumor (primitive or recurrence) with preservation of the healthy ovarian parenchyma and a lower risk of intraoperative rupture.

Methods: Between 2016 and 2018, 7 women with sonographic suspicion of borderline ovarian tumor ( 6 relapses and a first diagnosis) were referred to our Institution and treated with conservative surgery and intraoperative ultrasound. The average cysts diameter was $27 \mathrm{~mm}$ (range 16-45 mm). During laparoscopic surgery a laparoscopic linear ultrasound probe was introduced in one of the ancillary accesses, with exact location of the cyst.

Results: The average age of patients was 28; all patients except one were nulliparous and all were referred for a conservative treatment.

In 5 cases, intraoperative ultrasound enabled only enucleation of the cyst to be performed while preserving the ovary. In 2 cases a monolateral adnexectomy was performed: in one case intraoperative histological examination showed the presence of invasive tumor associated with borderline; in the other case during surgery a multifocal recurrence with poor identifiable healthy ovarian parenchyma was highlighted. In no case a cyst rupture occurred.

Patients underwent clinical follow-up with ultrasound and CA125 serial evaluation and after a mean follow-up period of 12 months (range 6-36 months) no patient experienced disease recurrence and one patient became pregnant.

Conclusions: Intraoperative ultrasound is a simple technique that allows to identify and enucleate adnexal cysts reducing the risk of intraoperative rupture and preserving healthy ovarian parenchyma in patients wishing to preserve fertility. 